\title{
Battling Against COVID-19 Infodemic in Indonesia: A Sociocybernetics Perspective
}

\section{Lovira L. Putri*1}

University of British Columbia, Canada

\author{
Reno F. Rafly \\ Catalyst Global Consulting, Indonesia
}

\begin{abstract}
As Indonesians collectively fight against the COVID-19 pandemic, the nation is simultaneously combatting the rampant spread of misinformation related to COVID-19. This phenomenon is often referred to as an 'infodemic,' defined by the World Health Organization (WHO) as the mass spread of information, factual or nonfactual, during a disease outbreak. In this article, we employ the methods of sociocybernetics analysis to examine the COVID-19 infodemic in Indonesia. We divide this paper into two sections. In the first section, we lay out the current state of the problem in Indonesia -how misinformation has challenged the post-pandemic recovery and changed the dynamics of Indonesian society at all levels, ranging from individuals to the society as-a-whole. In the second section, we propose a model, based on the approach of sociocybernetics, by which we propose to assess this challenge not just as a single entity but as a continuous, looping process, from the conception to the impact it has caused at all levels (micro, meso, and macro) of society. Given the complexity of this issue, we propose to develop an awareness and the education of cybernetics or systems thinking across multiple sectors when dealing with the infodemic in Indonesia.
\end{abstract}

\section{Key Words}

Infodemic, social media, system thinking, critical thinking, cybernetics, sociocybernetics, Covid-19

\footnotetext{
"Corresponding author:

Lovira R.Putri

University of British Columbia, Canada

Email: lovira@student.ubc.ca
} 


\section{Introduction}

The COVID-19 pandemic has brought a significant change in society. A technology change is no exception since we live in an era where we are more connected than ever. In fact, according to the World Health Organization (WHO), this is the first pandemic in recorded history where information is being spread at this rate (World Health Organization 2020). With the increasing channel of information flow, the truth-value of the content of the information itself becomes questionable. It is easier for any kind of information, factual or not, to be spread around by individuals or groups of individuals. In times where global health is in crisis, nonfactual information regarding the pandemic might turn into a much bigger problem. WHO classified this phenomenon as infodemic, namely, "(having) too much information including false or misleading information in digital and physical environments during a disease outbreak" (World Health Organization 2020).

Indonesia is home to 171.2 million internet users, according to a 2019 survey by the Indonesian Internet Service Providers Association (2019:3). It is not surprising that Indonesia is facing a major COVID-19 infodemic problem, on top of the pandemic itself. According to the Indonesian Ministry of Communication and Informatics, from January 2020 to September 2020 alone, 1984 COVID-19 hoax posts were found on social media (2020: 2).

The existential threat posed by the pandemic, coupled with the abundance of information related to the virus, creates a perfect storm that hinders the effort to recover from the COVID-19 pandemic. As such, for Indonesia to gear up towards post-pandemic recovery, it is essential for us as a society to combat the spread of the COVID-19 infodemic, alongside the pandemic itself.

\section{COVID-19 Infodemic in Indonesia}

The COVID-19 pandemic has brought significant changes to all aspects of society, including the relationship between society, technology, and information. One of the recommended measures enforced to reduce the spread of COVID-19 is to minimize physical contact with other people. To reduce physical contact between individuals, the Indonesian government issued Large Scale Social Restrictions (Pembatasan Sosial Berskala Besar) in some regions where there is an increase in the rate of COVID-19 cases. This restriction takes form in the closure of public spaces such as schools, offices, places of worship, and other places that facilitate public gatherings. This restriction induces changes in the way people interact with each other as these spaces typically facilitate such interactions and communications. 
It is no surprise that the abrupt change in life due to COVID-19 restrictions has brought negative impacts to the mental health of the people. Sutarto et al (2020) conducted a study using the Depression, Anxiety and Stress Scale (DASS-21) and found that of 472 Indonesian workers, 81.6\% experienced minimal to mild acute depression, 35.4\% experienced and $86.9 \%$ experienced stress. A nationwide study conducted by Andindyajati et al. (2020) found an increase in the anxiety level of Indonesians during the COVID-19 pandemic, which sits at 20\%, compared to $9.8 \%$ in 2018 . The majority of the respondents of the study were actively observing social restriction protocols. As such, it can be postulated that the social interaction restriction negatively impacted people's wellbeing.

We are witnessing and experiencing a global health crisis where technology and digital media are interwoven into our lives as it has never been before. With the role it has in our society, it is no doubt that technology and digital media could be one of the key contributors to the process towards COVID-19 recovery. The implementation of telemedicine, for example, eliminates the need for physical interaction when seeking medical care or consultation, which could reduce the spread of the virus (Bahl et al. 2020). Technology also has enabled researchers and scientists to share their latest COVID-19 research findings to the scientific and medical communities as well as the general public.

Social media is another example of technology that has been central during the COVID-19 pandemic. One way to define social media is "Internet-based channels that allow users to opportunistically interact and selectively self-present, either in real-time or asynchronously, with both broad and narrow audiences who derive value from user-generated content and the perception of interaction with others" (Carr and Hayes 2015: 50). Social media is no longer foreign for the majority of people in the world; one in three people in the world use social media (Ortiz-Ospina 2019). While there are numerous social media platforms available, Facebook, Instagram and WeChat are the three platforms with the greatest number of users (Ortiz-Ospina 2019).

In places where state-mandated lockdowns are enforced, social media provides an alternative means for people to connect and socialize with each other. Social media can also facilitate communication when the information evolves quickly (Gottlieb and Dyer 2020). On social media platforms, the exchange of information can go both ways by which a user can receive as well as give information that can be accessible to other individuals or a group of individuals. Once a post has been made by an individual, others may propagate it through actions such as sharing, liking, or commenting 
on the post, which could exponentially increase the spread and reach of such information (Buchanan 2020). The possibility to expose any kind of thoughts to a huge number of people, paired with limited regulation social media platforms currently have, creates a perfect vessel for information to rapidly spread across communities, regardless of whether or not such information is factual.

During the COVID-19 pandemic, there has been a reported trend in increasing screen-time in several countries around the world (Carroll et al. 2020; Hu et al. 2020; Ozturk and Yalçin 2020). Thus, we can expect that COVID-19 related content is being exchanged by people on social media. It is not a strange phenomenon. Even before the pandemic, people have been turning to social media for health-related information (CuelloGarcia et al. 2020). However, the credibility of some information found on social media is questionable. For example, more than a quarter of the most viewed COVID-19 related videos, which in total have 62 million views, contain non-factual information (Li et. al. 2020:).

Angeline et al. (2020) analysed 534 Indonesian articles that were classified as misinformation by the Indonesian Ministry of Communication and Information. The findings show that the majority of misinformation is commonly found in a form of social media text (41\%), followed by social media pictures $(31 \%)$ and social media video (17\%). The research also found that the most common content of misinformation in Indonesia is related to community spread, followed by public authority actions or policies.

The prevalence of misinformation during the COVID-19 pandemic in Indonesian society is a problem that affects every layer of society for information has the potential to snowball into bigger problems that go beyond the level of individuals. Next, we will review the potential challenges in three different levels of groups - micro, meso, and macro (Figure 1). 


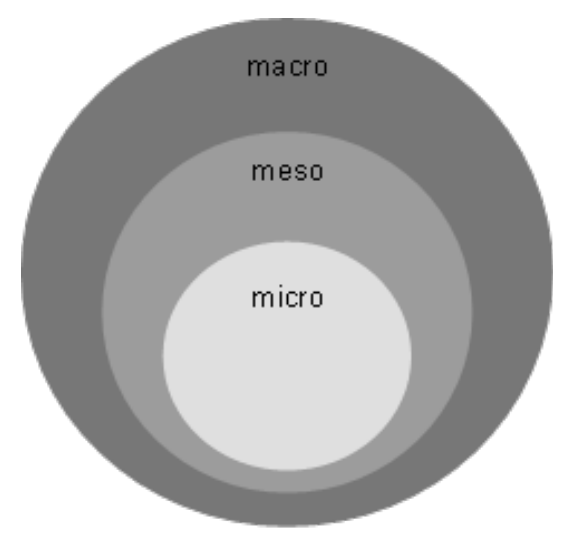

Figure 1. Three levels of groups - micro, meso, and macro

\section{Micro}

One of the direct impacts of infodemic is that constant exposure to COVID-19 news might be overwhelming to an individual, which can trigger stress, anxiety, or other mental health problems. There has been an extensive body of research that discovers the positive relationship between the information overload COVID-19 and stress and anxiety levels (Lee et al. 2020; Bendau et al. 2020; Mongkhon et al. 2021). The rise of stress and anxiety levels poses a risk to individuals as stress has been reported to weaken the immune system (Glasser and Kiecolt-Glaser 2005).

Misinformation might also lead one to follow medically inaccurate advice that has the potential to worsen the health of an individual. One of the types of misinformation found circulating is about medical advice related to COVID-19 (Angeline et al. 2020). Medical advice misinformation can range from less severe advice of gargling with warm salt water to a more severe and possibly life-threatening advice such as using the anti-malaria drug, hydroxychloroquine to cure COVID-19, which has been reported to cause serious heart rhythm problems and other safety issues (U.S. Food and Drug Administration 2020).

At the micro-level, misinformation can directly impact individuals, depending on the content of the information. Individuals who are exposed to the misinformation may then spread this news to their peers or family, regardless of whether such individuals believe in the information, furthering the harm to a broader level. 
Meso

At the meso level, the spread of misinformation creates a problem in communities or organizations within the Indonesian society that may slow down the process towards recovery. To halt the spread of the virus, a community effort is needed to reach the common goal that is to end the spread and recover from the pandemic. Information on social media platforms can spread from individuals to individuals, or from individuals to a group of individuals. Organizations often rely on a platform that can facilitate group communications, one common example used in Indonesia is group chat features on platforms such as WhatsApp, which is commonly used with families, neighbourhood communities, work, or school groups. Group chats could increase the reach and the speed of the spread of information, including non-factual information. When misinformation has reached the meso level, misinformation spread becomes harder to contain. The spread of misinformation at a community level has led to some grassroots efforts by the public, creating a public community that aims to battle misinformation on the internet such as MAFINDO (Masyarakat Anti Fitnah Indonesia).

\section{Macro}

As previously discussed, there is a positive correlation between the use of social media, which is rampant with misinformation, and the anxiety levels of individuals. If left untreated, the high level of anxiety that infodemic has caused may result in another public health crisis that the nation will have to face, during or post-pandemic. Some content of misinformation may contain health advice that could lead to hospitalization. As such, at a macro scale, infodemic could burden the already overwhelmed healthcare system.

Another effect of COVID-19 infodemic on the macro level is that it could trigger panic buying in a society. Some COVID-19 related misinformation contains the recommendation to use a certain product which was thought to reduce the spread of covid. Some of the items do help to reduce the spread of COVID-19 such as masks or other protective equipment. As the supply of these items is limited, panic buying could lead to the shortage of such equipment in society.

Recovery from COVID-19 requires all parts of society to work in tandem. To contain the virus, we also have to pay attention to the social and behavioural aspects that may influence the spread of the virus. In the previous section, we have described the COVID-19 infodemic in Indonesia 
and how it influences Indonesian society at all scales. In the next section, we will introduce our proposed model to combat the infodemic problem in Indonesia, borrowing concepts from (socio)cybernetics.

\section{Cybernetics}

The term Cybernetics originated from the Greek word kubernetes, meaning a steersman. Kubernetes was used by Plato to mean "the steering of people ", which later evolved into the modern-day "Governor". Cybernetics as a field of inquiry was pioneered by the Mathematician Norbert Wiener (1948) who defined it as "The science of communications and automatic control systems in both machines and living things." The act of governance requires some degree of control. Thus, cybernetics can also be understood as a principle of control that can be applied to any organized system (Herring and Kaplan 1998).

\section{Components of a Cybernetic System}

One important thing that lies at the heart of cybernetics is a cybernetics analysis concerns a system, of any scales, natural or artificial, where a system can be defined as "a whole consisting of interdependent components." (Hornung 2005: 8). Parts of a system make up a whole, functional unit. Generally, a system consists of an input, a processing unit, an output, and the environment. The environment, in this case, is everything outside of the system. This structure can manifest differently depending on the application, but any system can be abstracted in this sense.

It is important to note that not all systems are cybernetic systems. Systems theory or science, although it may seem similar, is different from cybernetics. Both are analyses of systems. General Systems Theory typically makes inquiries about the common laws that exist within any kind of system (Bertalanffy 1968), where cybernetics emphasizes the goalorientedness and functional control of a system. Heylighen and Joslyn (2001:3) said:

Perhaps the most fundamental contribution of cybernetics is its explanation of purposiveness, or goal-directed behavior, an essential characteristic of mind and life, in terms of control and information. 
A goal, or a purpose, is an important concept in cybernetics. A goal can take on many forms depending on the system itself. As a goal-oriented system, a cybernetic system will direct itself to achieve its goal, and when the system deviates away from the goal, it corrects itself by minimizing the difference between the current state and the goal. This act of minimizing the difference between the goal and its current state can be thought of the steering, governance, or control that characterizes a cybernetic system.

To know the difference between a system's current state and the goal, a cybernetic system utilizes what is known as a feedback loop. This is another defining feature of a cybernetic system, that it is a closed-loop system. The output of the system circulates back to the system itself. A feedback loop enables a system to regulate itself - to use the output as a guide to control the system to bring it closer to the desired state or the goal.

In other words, information about the output and the changes that the output and the environment brings to the state of the system travels back into the system itself. The information will then use the information it receives to govern the system to bring it closer to the goal.

\section{First-Order vs Second-Order Cybernetics}

In general, cybernetics as a field is distinguished into two kinds, the first is the initial formation of cybernetics and the second is the updated view as a result of the maturation of the field. At the beginning of its conception, the cybernetics approach studies a system from an outside perspective. It is the "study a system as if it were a passive, objectively given "thing", that can be freely observed, manipulated, and taken apart" (Heylighen and Joslyn 2001:3). The observer of the system is, in this case, separate from the system itself.

Second-order cybernetics, while the essence of the inquiry remains the same, it distinguishes itself from its predecessor by adding the observer into the equation. Rather than looking at a system from an outsider's point of view, the observation is made from within (Figure 2). Thus, the observer is also being observed - there is a self-referential aspect of this observation. Second-order cybernetic assesses a system as "an agent in its own right, interacting with another agent, the observer. As quantum mechanics has taught us, observer and observed cannot be separated, and the result of observations will depend on their interaction" (Heylighen and Joslyn 2001:4).

As Von Foerster, one of the pioneers of second-order cybernetics, 
puts it, first-order cybernetics is the study of "observed systems" while second-order cybernetics is the study of "observing systems" (Hornung 2005). Observers are not neutral but engaged with the system. The way the observers think about the way they think matters and will affect the system it observes.
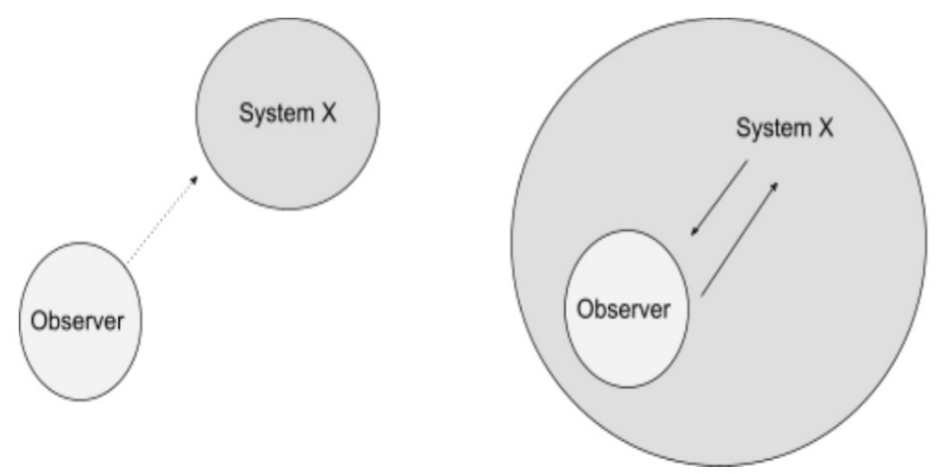

Figure 2. First-order cybernetics (left) Second-order cybernetics (right)

\section{Sociocybernetics}

Modern social problems require solutions to deal with their growing complexity. Social problems live in a society, which can be thought of as a system of sub-systems that interrelate with one another.

Sociocybernetics can be defined as the application of systems science and first and second-order cybernetics to sociology and other social sciences (Hornung 2005). Sociocybernetics combines concepts from sociology as well as cybernetics, such as first/second-order observation distinction, systems approach, and feedback loops, applying it to social systems.

Sociocybernetics emphasizes the self-steering of social groups and not deterministic control of social groups (Almaguer-Kalixto and Giglietto 2019). Self-steering takes place as a combination of both feedback and feedforward loop. Feedback, as described in the previous section, controls a system using the difference between the goal and the output. On the other hand, feedforward systems utilize forward-thinking, proactively anticipating errors that may disturb a system (Figure 3). 


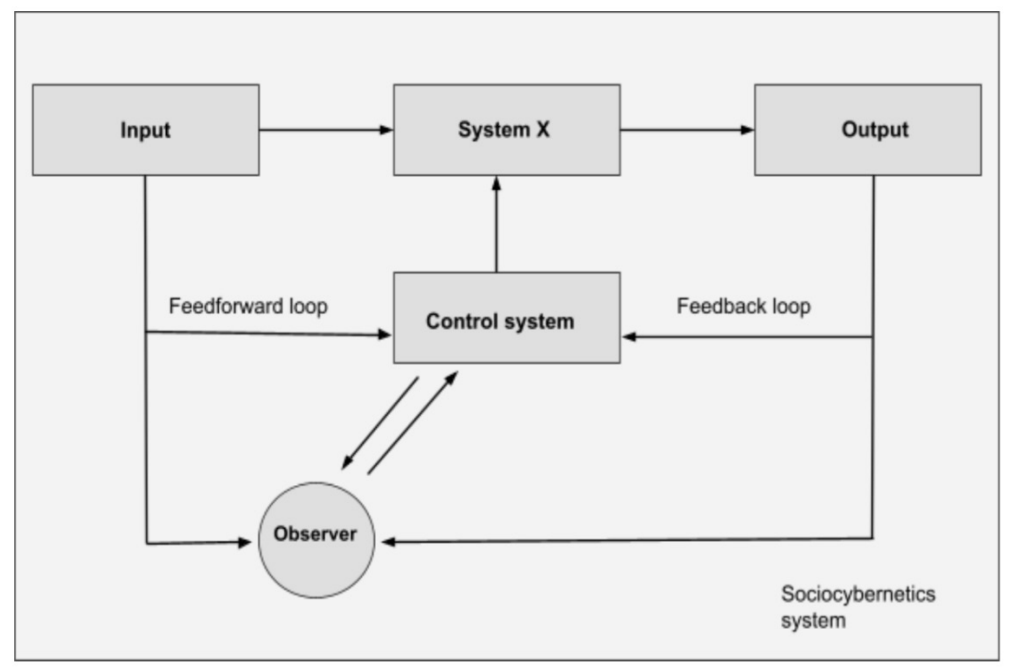

Figure 3. Sociocybernetics System

\section{Our Proposed Model: How Sociocybernetics Approach Can Help Reduce Infodemic}

Using the concept of the sociocybernetics model, we propose to combat the infodemic by looking into how we can empower individuals or organizations to use a closed-loop system in information processing. There are two goals of using sociocybernetics as follows.

\section{To Reduce the Transmission of Misinformation}

The use of critical thinking can lessen the transmission of misinformation. According to Paul and Elder (2006), there are two levels of thinking (Figure 4):

a. First-order thinking, which is the kind of thinking that is unfiltered, impulsive, and spontaneous. This kind of thinking is quick, easy, and requires very little thinking skill. It is what most people do when they first receive any information. However, it is prone to bias and error and can increase the level of quick transmission.

b. Second-order thinking, which adds a process into our thinking, checks in on our assumptions and analyses other information that might be pertinent before transmitting the information or making a decision. This is what we call critical thinking, where we become a critic of our thinking. 
When an individual or organization is equipped with critical thinking, the amount of transmission of misinformation can be significantly reduced and prevent further misinformation that can be fatal. By thinking critically, it means we align ourselves to use the sociocybernetics approach.

Within the sociocybernetics model, if we can empower an individual, a group, or an organization with critical thinking, in the long run, they can gain the information, analyse it and make informed decisions on what to do and what to improve next time. The closed feedback loop system will create a self-governed organization where they can review themselves and gain the feedback to self-correct themselves. This system thinking will also increase the level of positive change management to empower themselves to make their improvements and to create better solutions next time. If done correctly, managing information using a sociocybernetics system can increase the ability of an organization to gain innovation.

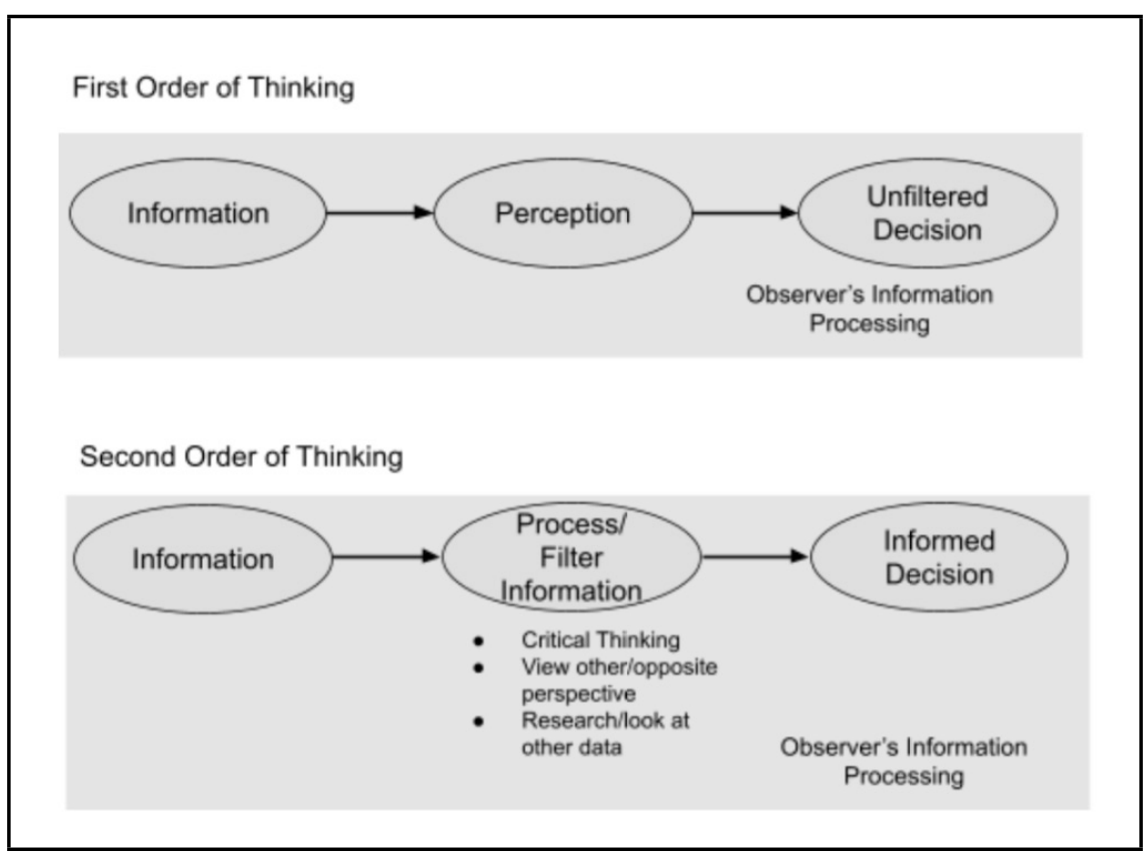

Figure 4. First Order and Second Order of thinking

\section{To Reduce the Impact Caused by the Misinformation}

Sociocybernetics can also help reduce the impact that is caused by misinformation. When an individual or an organization adds critical thinking when processing information, they will automatically pause and filter the information using several methods, for example, by adding to 
view other perspectives or opposite viewpoints, checking whether the information is legit, and conducting further research.

An example of misinformation is the spread of "fake news" of vaccination. In January 2021, there was misinformation circulated across chat groups about vaccination containing religiously forbidden (haram) ingredients (Ningtyas 2021). Given Indonesians are predominantly Muslims, news like this will greatly impact the speed of people getting a much-needed vaccination to boost their immune system and to reduce the spread of Covid-19.

Using a sociocybernetics approach, groups and organizations can inoculate themselves against this type of misinformation by conducting information processing, thinking and assessing, challenging assumptions, and using the right information to fight it back (Hornung 2005). This approach can empower groups to have the ability to think critically and make informed decisions either to respond or to stop further spread. Over time, using the same approach, they will be able to combat other future misinformation and become informed citizens.

Another example of misinformation was captured by a survey conducted by the University of Indonesia that revealed $21 \%$ out of 772 respondents in greater Jakarta believed that coronavirus is a conspiracy (The Jakarta Post 2020). This will impact vaccine hesitancy if not managed well and can further challenge the fight against Covid-19.

The Indonesian government has conducted several programs and initiatives to combat hoaxes and the spread of misinformation. One of them is to establish a website page "Laporan Isu Hoaks" via their website kominfo.go.id. Another program is to establish a cybercrime division under Indonesia Police Force (POLRI) to combat fraud, cyber fraud, and cybercrime. These moves are notably going in the right direction. Furthermore, we propose using the sociocybernetics approach in proactively educating groups and citizens through multiple platforms to build a solid foundation in our citizens in combating misinformation, especially during the COVID-19 pandemic.

\section{Conclusion}

This article has shown that battling against the COVID-19 infodemic in Indonesia needs multiple approaches. One of them is to build a solid foundation of a closed feedback loop system using a sociocybernetics approach so that individuals, groups, and organizations can use the 
feedback to conduct a self-governance and self-correction in fighting against misinformation. By doing so, the ecosystem will be able to use the right approach according to their needs and contexts in building the right filter to assess, analyse, and make informed decisions.

We recommend that organizations and government proactively design helpful methods in combating misinformation that need to be included in future programs and policies. At the micro (individual) level, we propose to equip individuals by introducing critical thinking concepts beginning in early education. Students as early as elementary school years can learn what to do when they receive new information, analyse the information, ask a trusted person about the information, and make an informed decision. Early exposure to massive information through social media has the negative potential to alter their thinking and decision-making process permanently. By providing this skillset early on, we can help strengthen their thought process, understand that "not all you see in social media are true", and educate them to analyse the information before sharing.

At the meso level, communities and organizations can build their feedback loop system through a channel where people can report and assess incoming information to check whether it is legit and coming from a reputable source. In addition, everyone should be trained not to automatically "forward" or "share" any incoming information to chat groups and social media before making sure the accuracy and validity of the information. Peer-checking information can also help when people in the group are encouraged to speak up and ask if there is any information, rumours, or news that may seem misleading or damaging to a certain extent. Regular awareness should be provided from time to time so that, over time, it becomes a habit and norm that is built permanently into the community and the organization.

Lastly, at the macro level, in making public policy, the key is to proactively combat misinformation through ongoing public awareness via multiple platforms such as social media and chat groups as well as providing training through local communities and local PKK (Pemberdayaan \& Kesejahteraan Keluarga) about the danger of misinformation and how to increase self and group awareness in combating against it. In addition, the government should partner with social media platforms to help flag ambiguous data or any potentially harmful information while reducing the virality of hoaxes. News media can also help by sharing accurate information and positioning themselves as one of the valid sources the public can rely on. By doing this, we can build reliable sources and reduce the power of unreliable sources going into the public system. 
In conclusion, by building a solid capability in critical thinking and using effective feedback in sociocybernetics, we can have a better strategy in battling against the infodemic in Indonesia. While the above approaches may take some time to have a notable impact, however, in the long run, as a nation we will be better prepared in fighting against misinformation and will be better equipped with better tools. By empowering them through critical thinking skills and building the right capabilities to conduct the right feedback response and self-governance, over time our nation will be much stronger in battling against infodemic.

\section{Funding}

The authors received no financial support for the research, authorship, and/ or publication of this article.

\section{References}

Almaguer-Kalixto, Patricia Eugenia, and Fabio Giglietto. 2019.

"Steering the World from Where We Are: An Introduction to the Sociocybernetics Perspective." Current Sociology 67(4):479-494.

Angeline, Mia, Yuanita Safitri, and Amia Luthfia. 2020. "Can the Damage

Be Undone? Analyzing Misinformation during COVID-19

Outbreak in Indonesia." The 2020 International Conference on

Information Management and Technology (ICIMTech): 360-364.

Anindyajati, Gina et al. 2021. "Anxiety and Its Associated Factors during the Initial Phase of the COVID-19 Pandemic in Indonesia." Front Psychiatry 12, Mar 10.

Asosiasi Penyelenggara Jasa Internet Indonesia. 2020. "Laporan Survei Internet APJII 2019-2020 (Q2)”. Retrieved June 28, 2021 (https:// www.apjii.or.id/content/read/39/521/Laporan-Survei-InternetAPJII-2019-2020-Q2).

Bahl, Shashi et al. 2020. "Telemedicine Technologies for Confronting

COVID-19 Pandemic: A Review." Journal of Industrial

Integration and Management 05(04):547-561.

Bendau, Antonia et al. 2020. "Associations between COVID-19 Related Media Consumption and Symptoms of Anxiety, Depression and COVID-19 Related Fear in the General Population in Germany." European Archives of Psychiatry and Clinical Neuroscience 271(2):283-291. 
Buchanan, Tom. 2020. "Why Do People Spread False Information Online? The Effects of Message and Viewer Characteristics on Self-Reported Likelihood of Sharing Social Media Disinformation." PLOS ONE 15(10).

Carr, Caleb T., and Rebecca A. Hayes. 2015. "Social Media: Defining, Developing, and Divining." Atlantic Journal of Communication 23(1):46-65.

Carroll, Nicholas et al. 2020. "The Impact of COVID-19 on Health Behavior, Stress, Financial and Food Security among Middle to High Income Canadian Families with Young Children." Nutrients 12(8):23-52.

Cuello-Garcia, Carlos, Giordano Pérez-Gaxiola, and Ludo van Amelsvoort. 2020. "Social Media Can Have an Impact on How We Manage and Investigate the COVID-19 Pandemic." Journal of Clinical Epidemiology 127:198-201.

Glaser, Ronald and Janice Kiecolt-Glaser. 2005. "Stress Damages Immune System and Health." Discovery Medicine 5(26).

Gottlieb, Michael and Sean Dyer. 2020. "Information and Disinformation: Social Media in the COVID-19 Crisis." Academic Emergency Medicine 27(7):640-641.

Herring, Charles and Simon Kaplan. 1998. "Cybernetic Components: A Theoretical Basis for Component Software Systems." Component Oriented Software Engineering Workshop (COSE '98).

Heylighen, Francis, and Cliff Joslyn. 2001. "Cybernetics and SecondOrder Cybernetics." Encyclopedia of Physical Science and Technology 155-69.

Hornung, Bernd R. 2005. "Principles of Sociocybernetics." European System Science Union (ESSU) 6th Congress, Symposium of Sociocybernetics.

Hu, Zhao, Xuhui Lin, Atipatsa Chiwanda Kaminga, and Huilan Xu. 2020. "Impact of the COVID-19 Epidemic on Lifestyle Behaviors and their Association with Subjective Well-Being among the General Population in Mainland China: Cross-Sectional Study." Journal of Medical Internet Research 22(8): e21176.

Lee, Jung Jae et al. 2020. “Associations between COVID-19 Misinformation Exposure and Belief with COVID-19 Knowledge and Preventive Behaviors: Cross-Sectional Online Study." Journal of Medical Internet Research 22(11): e22205. 
Mongkhon, Pajaree et al. 2021. "Exposure to COVID-19-Related Information and Its Association with Mental Health Problems in Thailand: Nationwide, Cross-Sectional Survey Study." Journal of Medical Internet Research 23(2): e25363.

Ningtyas, Ika. 2021. "Indonesia Battles Spread of Vaccine

Misinformation". Anadolu Agency. Retrieved June 26, 2021

(https://www.aa.com.tr/en/asia-pacific/indonesia-battles-spreadof-vaccine-misinformation/2124319).

Ortiz-Ospina, Esteban. 2019. "The Rise of Social Media." Our World in Data. Retrieved June 29, 2021 (https://ourworldindata.org/rise-ofsocial-media).

Ozturk, Aslihan and Aylin Yalçin. 2021. "Relationship between Parenting Practices and Children's Screen Time during the COVID-19

Pandemic in Turkey." Journal of Pediatric Nursing 56:24-29.

Paul, Richard W. and Linda Elder. 2002. Critical Thinking: Tools for Taking Charge of Your Professional and Personal Life. Upper Saddle River, NJ/USA: Financial Times Prentice Hall.

Sutarto, Auditya Purwandini, Shanti Wardaningsih, and Wika Harisa Putri. 2021. "Work from Home: Indonesian Employees' Mental Well-Being and Productivity during the COVID-19 Pandemic." International Journal of Workplace Health Management 14 (4): 386-408.

The Jakarta Post. 2020. "Some Indonesians Still Believe Covid-19 is a Conspiracy: A Survey.” Retrieved June 22, 2021 (https://www. thejakartapost.com/news/2020/11/08/some-indonesians-stillbelieve-covid-19-is-a-conspiracy-ui-survey.html).

U.S Food and Drug Administration. 2021. "FDA Cautions Use of Hydroxychloroquine/Chloroquine for COVID-19." Retrieved June 25, 2021 (https://www.fda.gov/drugs/drug-safety-andavailability/fda-cautions-against-use-hydroxychloroquine-orchloroquine-covid-19-outside-hospital-setting-or).

Von Bertalanffy, Ludwig.1968. General System Theory: Foundations, Development, Applications. New York, NY: George Braziller.

Wiener, Norbert. 1948. Cybernetics or Control and Communication in the Animal and the Machine. Cambridge, MA: MIT Press.

World Health Organization. 2021. "Infodemic.” Retrieved June 21, 2021 (https://www.who.int/health-topics/infodemic \#tab=tab_1). 